\title{
Take-home laboratory for a course in radiometry
}

\section{James Palmer}

James M. Palmer, "Take-home laboratory for a course in radiometry," Proc. SPIE 2525, 1995 International Conference on Education in Optics, (13 October 1995); doi: 10.1117/12.224021

SDIE Event: SPIE's 1995 International Symposium on Optical Science, Engineering, and Instrumentation, 1995, San Diego, CA, United States 


\title{
Take-home laboratory for a course in radiometry
}

\author{
James M. Palmer \\ Optical Sciences Center, University of Arizona \\ Tucson, AZ 85721
}

\begin{abstract}
A set of take-home experiments associated with a junior-level course entitled Radiometry, Sources and Detectors has been developed. The students are furnished with a small, inexpensive radiometer and a kit of accessories which allow a wide variety of self-conducted experiments to be performed. A laboratory manual has also been developed to guide the student through a series of eleven experiments. The student must prove radiometric concepts in several cases, and must develop the details of the experiment. This paper describes the concept, the apparatus and the experiments, and reports on the results.
\end{abstract}

KEYWORDS: education, radiometry, laboratory

\section{INTRODUCTION}

The subject of radiometry, the measurement of optical radiation, is usually taught as a lecture course. In this teacher's opinion, any course involving measurement should offer hands-on experience in the form of an associated laboratory. However, space, time and financial constraints frequently prohibit the offering of a formal laboratory course or even a significant component within another laboratory course. This paper describes a set of take-home experiments associated with a junior-level course in Optical Engineering entitled Radiometry, Sources and Detectors. The students are furnished with an inexpensive but versatile radiometer and a kit of accessories which allow a wide variety of self-conducted experiments to be performed. A set of experiments demonstrates the way a radiometer works, illustrates important radiometric principles, and facilitates the radiometric characterization of our local environment. An accompanying laboratory manual has also been developed to guide the student through the series of eleven experiments. An important feature is that in several cases, the student is simply asked to prove a radiometric concept, and must work out the details of the experiment, thereby discovering the inherent problems and traps. The lab was developed for an undergraduate BSOE program but is readily adapted for use with a graduate level course. The article by Johnston and Schroeer (1992) was particularly helpful.

\section{THE LABORATORY KIT}

The student is furnished a working radiometer, the VERSA-350, complete with two fresh $9 \mathrm{~V}$ alkaline batteries and a complement of lenses, filters, etc. We also furnish a breadboard and parts kit for operational-amplifier (op-amp) and detector interfacing experiments. The entire kit and caboodle (Figure 1) is housed in an attractive green military surplus ammunition canister that is both rugged and watertight; the student who possesses one of these presents a unique and distinctive appearance on campus. 


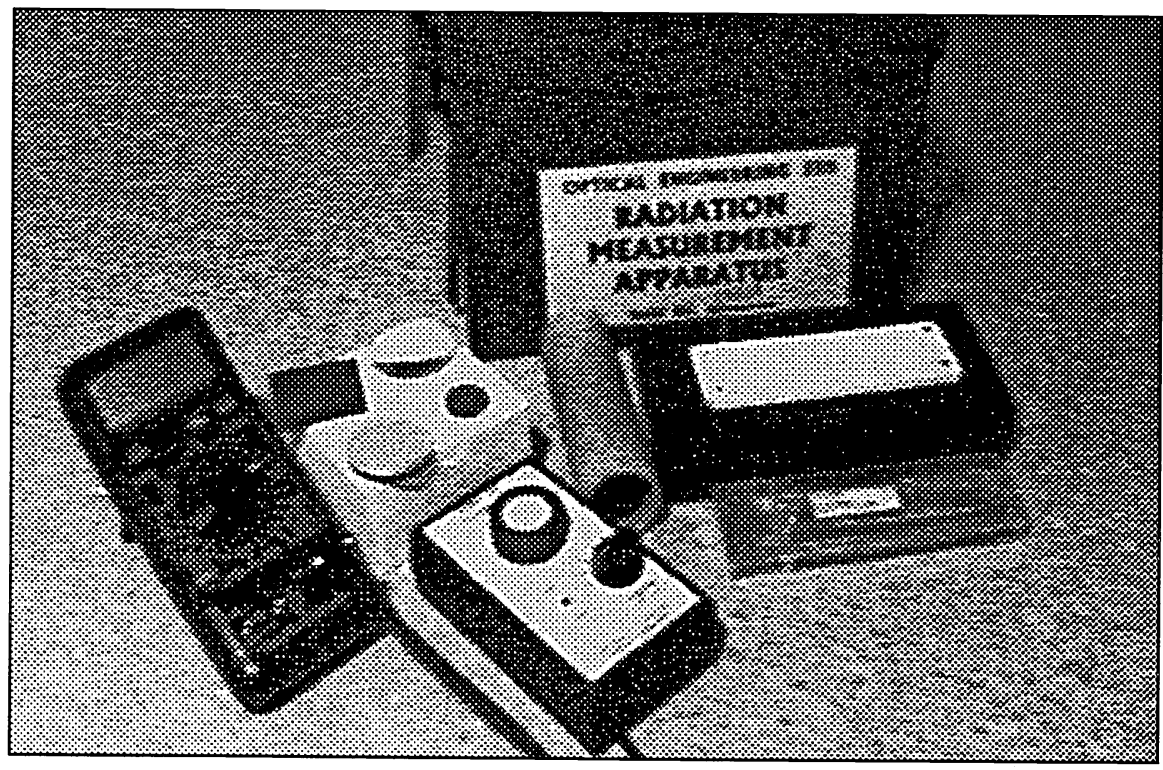

Figure 1

Portable radiometry laboratory

The $\boldsymbol{V E R S A - 3 5 0}$ radiometer (Figure 2) was designed as a multi-purpose instrument to demonstrate fundamental radiometric principles. It includes two detectors (plug interchangeable), one a photovoltaic (PV) silicon ( $\mathrm{Si}$ ) device responding in the visible and near- $\mathbb{R}$ regions of the optical spectrum, and the other a photoconductive (PC) cadmium sulfide $(\mathrm{CdS})$ device with response restricted to the visible. A black anodized aluminum ring allows mounting accessory filters, diffusers, tubes, etc. The electronics, which are mounted on a custom etchedcircuit board, provides bias for the PC detector and for the PV detector when reverse-biased. The transimpedance amplifier (TIA) has normal ranges from $10^{3}$ to $10^{7}$ and features a TEST position that can be used for an additional experimental gain component (such as an integrating capacitor) or for assessing the health of the radiometer. All internal gain resistors are $1 \%$ metal film. The radiometer is powered by two internal $9 \mathrm{~V}$ alkaline transistor batteries and the internal supplies are regulated to $\pm 5 \mathrm{~V}$. The output cable plugs into any standard DMM with banana jacks ( $0.75^{\prime \prime}$ spacing.)

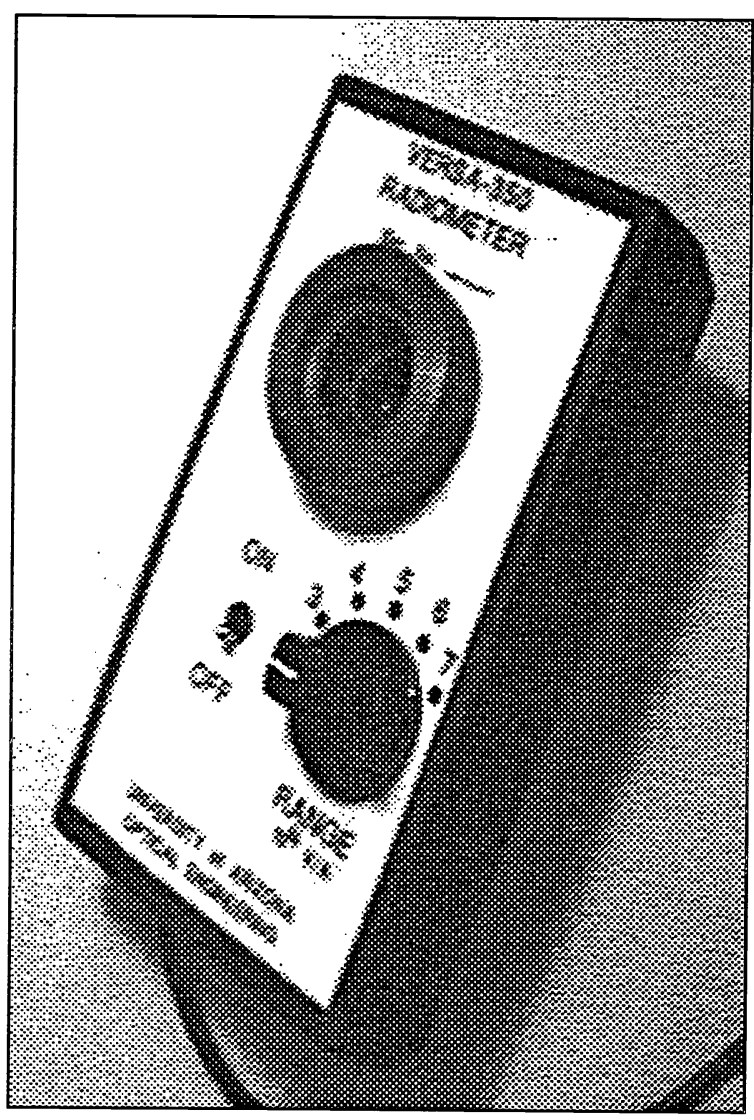

Figure 2. The VERSA-350 Radiometer 
A schematic diagram of the internal electronics is shown in Figure 3. The range switch controls the feedback resistor of the TIA. The numbers 3 through 7 on the range switch represent the exponent $\mathrm{N}$ for the transimpedance gain, i.e. the resistance is $10^{\mathrm{N}}$ ohms, and the transfer function is $10^{\mathrm{N}}$ volts/amp. The radiometer is normally equipped with a $100 \Omega$ resistor in the test (T) position of the range switch, which is useful for the PC detector in bright light. It may be replaced with other values, or a test jumper may be changed for diagnostic purposes by the instructor or lab manager. The output of the $V E R S A-350$ is a voltage in the range 0$4 \mathrm{VDC}$, limited by the internal power supplies. The student is asked to acquire a small handheld digital multimeter with at least a $200 \mathrm{mV}$ full-scale (100 $\mu \mathrm{V}$ per division) range. There are many from which to choose; satisfactory models can be found at prices below $\$ 40$.

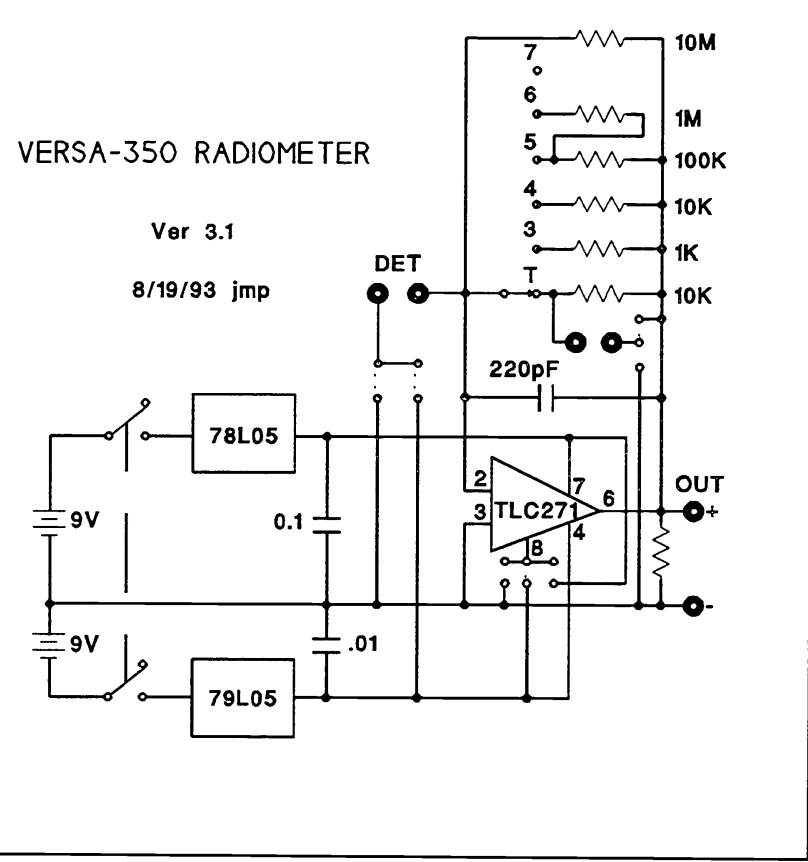

Figure 3. VERSA-350 schematic diagram

Internal access via two screws is required to test and change batteries and to interchange detectors. The batteries should be replaced when their voltage drops below 7V. An unbiased photovoltaic silicon detector is initially installed with the bias jumper located on jumper pins labeled "PV". To reverse-bias the Si detector, shift the jumper to the pins labeled 'PC'. The CdS detector is normally "parked" on two unused pins towards the edge of the board. When it is used, it will replace the Si detector, and the bias jumper is positioned at 'PC'. The radiometric accessories supplied with the VERSA-350 radiometer are listed in Table 1.

Table 1. Parts list for radiometry exercises

\begin{tabular}{|l|l|}
\hline Accessory attachment flange & Polarizers (2) \\
\hline Cardboard tube & White plastic diffuser \\
\hline Fuzzy-black paper for above & Visible-transmitting filter \\
\hline Lens, $\mathrm{f}=150 \mathrm{~mm}$ & Infrared transmitting filter \\
\hline
\end{tabular}


Three of the lab exercises deal with operational amplifiers and detector interfacing. To this end, a separate breadboard is furnished along with all of the necessary components. The breadboard is mounted on top of a compartmented anti-static container which houses the loose components. The parts supplied for the interfacing experiments are listed in Table 2; those with an RS- part number are available from Radio Shack.

Table 2. Parts list for detector interfacing experiments

\begin{tabular}{|c|c|}
\hline QTY & DESCRIPTION \\
\hline 1 & Plug-in breadboard (RS 276-174), mounted on storage box \\
\hline 2 & 9V battery, preferable alkaline (RS 23-553 or equivalent) \\
\hline 2 & Battery clips (RS 270-325) \\
\hline 1 & Jumper kit (RS 276-173) \\
\hline 2 & Op-amps (1 LM317 bipolar, 1 TIL0781 FET) \\
\hline 1 & 78L05 Regulator \\
\hline 7 & Capacitors $[10,100 \mathrm{pF}(2), 0.001,0.01(2), 0.1 \mu \mathrm{F}, 1 \mu \mathrm{F}]$ \\
\hline lot & Assorted resistors (RS 271-309) $+10 \mathrm{M} \Omega$ \\
\hline set & Test clips (RS 270-334) \\
\hline lot & Assorted LED's (Red, Green, Yellow, IR) \\
\hline 1 & Thermistor (RS 271-110) \\
\hline 1 & 1N34 Germanium diode (wide black cathode stripe) (RS 276-1123) \\
\hline 1 & 1N914 Silicon diode (narrow black cathode stripe) (RS 276-1122) \\
\hline
\end{tabular}

\section{OUTLINE OF THE LABORATORY SESSIONS}

The 11 lab sessions are shown in Table 3. A paragraph or two is devoted to each session to delineate the objectives and outline the procedures.

\begin{tabular}{c|l} 
LAB & TITLE \\
\hline 0 & Familiarization with the $\boldsymbol{V E R S A - 3 5 0}$ \\
1 & Geometrical Characterization of a Radiometer \\
2 & Radiance and its Invariance \\
3 & Inverse Square Law \& Cos ${ }^{\mathrm{n}}$ Laws \\
4 & Radiometric Calibration \\
5 & General Source Characteristics \\
6 & Radiometric Properties of Materials \\
7 & The Operational Amplifier - properties and circuits \\
8 & Interfacing PC Detectors \\
9 & Interfacing PV Detectors \\
10 & Polarization in Radiometry
\end{tabular}




\section{Lab 0. Familiarization with the VERSA-350}

This brief lab exercise is a later addition, designed to introduce the student to the hardware, how to use the $V E R S A-350$ and the DMM. It was added after a student went through an entire semester reading the radiometer output using current ranges on the DMM. It also vividly demonstrates saturation, the ultimate limit of linearity, and alerts the student that whenever all of the readings are equal even though the input conditions are changing, something is wrong! Finally, the lab instructs the optimum use of the range switches on the DMM and VERSA-350, and demonstrates the dynamic range of the system.

\section{Lab 1. Geometrical characterization of a radiometer}

In this extended lab, the student determines several geometric characteristics about the VERSA-350 radiometer to allow an understanding of the behavior of the instrument as well as the radiometric behavior of several interesting materials.

The first step measures the active area of the detector and the limiting aperture dimensions to determine the "nominal" field-of-view (FOV) of the bare radiometer. The results are confirmed by a direct measurement of the angular response using a small, bright light bulb and a homemade (cardboard and protractor) turntable. The relative response (normalized to unity at normal incidence) is recorded at angles from 0 to 85 degrees in 5 degree increments, with smaller increments where the signal is changing rapidly with angle. This is Irradiance Configuration I. Second, the solid white plastic diffuser is placed in the well and the angular response is again determined and compared with the results of the first measurements and with the ideal cosine response curve. This is Irradiance Configuration II. These two configurations are used for measuring irradiance (watts per unit area) received from a hemisphere.

The third step involves preparing a cardboard tube to place the furnished lens at its focal length (approximately $150 \mathrm{~mm}$ ) from the detector. We do the angular scan without the lens to demonstrate how a simple tube can establish a definitive field-of-view. The tube is then lined with a piece of fuzzy-black paper and the angular measurements repeated to demonstrate the contribution of stray light. This is Radiance Configuration I. Next, the lens is installed and the angular scan repeated to characterize Radiance Configuration II. Measurements are compared with calculated values based on measured physical dimensions. Peak normalization is computed on each of the four configurations for later use.

The final step places apertures of various sizes in front of the lens to demonstrate the effect of entrance pupil area on the output signal. In addition, an angular scan is done with one of these apertures to compare the FOV with the earlier scans.

\section{Lab 2. Radiance and its invariance}

This short lab has the student place the radiometer in Radiance Configuration II (tube and lens.) A potential uniform lambertian source such as a large, white sunlit wall is identified, and its radiometric properties explored. We prove that radiance is conserved by measuring the output of the radiometer as a function of distance between the wall and the radiometer. We then show that the source is quasilambertian by measuring the radiance as a function of angle.

The limits are explored by building a small lambertian source by placing a light bulb in a box with a white paper "window." The radiometer output is plotted vs. distance and interpreted with regard to the radiometer FOV. These measurements are done using three FOV configurations: (1) irradiance, (2) radiance with tube, and (3) irradiance with tube and lens. 


\section{Lab 3. Inverse square law $\& \cos ^{n}$ laws}

The challenge is to confirm the inverse square law and the $\cos ^{\mathrm{n}}$ laws using the VERSA-350 in Irradiance Configuration II (white plastic diffuser). For each of these confirmations, the student must design, build and verify the light source used, an isotropic source for the inverse square and $\cos ^{3}$ laws, and a lambertian source for the $\cos ^{4}$ law. The data obtained is plotted and a theoretical curve superimposed to demonstrate confirmation. A conventional flashlight is also used to illustrate that not all sources behave in an isotropic manner. The $\cos ^{\mathrm{n}}$ laws are verified over the range 0 to $60^{\circ}$. One of the main lessons learned is the identification and control of stray light. Beware the "white-shirt" effect!

\section{Lab 4. Radiometric calibration}

This extended exercise is considered both the most difficult and the most important of all. The result is a reasonable calibration for the $V E R S A-350$. One of the two filters supplied with the radiometer is a visible-transmitting, infrared-absorbing filter that, in combination with the Si detector, gives a crude approximation to the human eye response. The other filter blocks the visible and transmits the nearinfrared. Curves showing the relative spectral responsivity of the detector and the spectral transmission of the two filters are included. Prior to the lab, the student is asked to plot the eye response curve and the spectral responsivity of the radiometer, bare and with each filter, and do both peak and moments normalization.

The physical measurements are carried out in small groups in the authors' radiometric calibration laboratories. The first lamp used for calibration is an inexpensive luminous intensity standard with a rating of about 30 candelas and an effective temperature of $2700 \mathrm{~K}$. Using the visible-transmitting filter and the white plastic diffuser in front of the silicon detector, we measure the output of the radiometer at a distance of 1 meter. A calibrated photometer is also placed at one meter from the lamp on an optical bench for comparison. Using the rating of the light bulb and the assumption that the radiometer has a spectral response the same as the human eye, we can determine the radiometer responsivity in volts per lumen. Measurements are also made with the bare detector and with the infrared-transmitting filter to test the conceptual meaning of a photometric calibration.

Next we calibrate the bare Si detector using a He-Ne laser at a wavelength of $632.8 \mathrm{~nm}$. We determine the laser power with a calibrated Si detector just before the student uses it to measure their radiometer. Subsequent calculation steps include the determination of the responsivity in $\mathrm{A} / \mathrm{W}$ and the quantum efficiency at $632.8 \mathrm{~nm}$. This absolute calibration, along with the furnished relative wavelength response curve, allows the student to derive a curve of absolute spectral responsivity vs. wavelength.

The other radiometric calibrations take place in a black room using a 1000-watt quartz-halogen (type DXW) lamp on an optical bench This lamp is similar to those used for standard lamps, and the spectral irradiance data from the calibration certificate of a real calibrated lamp (operating current of 8 amps DC) is used to represent our lamp. The radiometer (Irradiance Configuration I) is placed at a known location on the bench and the output signal data (volts, range) is noted using the radiometer bare, with the visibletransmitting filter, and with the infrared-transmitting filter. Using the given relative spectral responsivity for silicon, we can determine the absolute spectral responsivity vs. wavelength and compare it with the value at $633 \mathrm{~nm}$ obtained with the He-Ne laser. Peak and moments absolute bandwidth normalizations on each detector combination, bare and filtered. The white plastic diffuser is then added (Irradiance Configuration II) to determine the loss incurred with the use of the diffuser. 
We determine the radiance responsivity using the same "calibrated" DXW source in conjunction with a Kodak gray card (reflectance $\rho=0.18$, assumed lambertian) at a distance of $150 \mathrm{~cm}$. The radiometer observes this target in both Radiance Configuration I and Radiance Configuration II and the results are compared to the irradiance calibration using the bare detector. We attempt to reconcile this calibration with the irradiance calibration using predetermined geometrical factors (area and FOV).

The final exercise is the determination of linearity via the superposition method. The suggested procedure is to shine one light source on the radiometer and note the reading. Shine a second source on it and note the second reading. Now shine them both on the radiometer simultaneously and note the third reading and compare the measured sum with the calculated sum. If the light sources are repeatable and the radiometer is linear, they should be equal. The linearity of the Si detector is demonstrate over several decades of output, over the current range from $10^{-8}$ to $10^{-3}$ amperes.

An EXTRA CREDIT exercise is to devise a method, knowing the effective temperature of the luminous intensity lamp lamp, to determine the peak responsivity of the bare $\mathrm{Si}$ detector, and scale the relative curve using this peak value.

\section{Lab 5. General source characteristics}

Irradiance Mode $I$ is first used with the bare radiometer and direct sunlight as the source. Measurements are next made with each of the two filters (visible-transmitting and infrared-transmitting.) We then repeat with skylight, a tungsten lamp and a fluorescent lamp. Using the absolute responsivity of the detector determined in Lab 4, we compute the in-band radiant power and irradiance from each source in the two spectral bands and draw conclusions as to the spectral content of the four sources.

Another interesting exercise measures the irradiance from the full moon and from the half-moon, also using Irradiance Mode I. Is the ratio between full moon and half moon 2:1? Should it be?

An EXTRA CREDIT exercise uses our new two-color radiometer (using the above filters) to determine the ratio temperature of direct sunlight, skylight and a tungsten lamp by comparing the ratio of VIS/IR with blackbody radiation at various temperatures. This requires computing the blackbody integrals over the wavelength responses of the radiometer as determined in Lab 4.

\section{Lab 6. Radiometric properties of materials}

Many common materials have interesting radiometric properties, including reflectance and transmittance. To make reflectance measurements we compare the surface being measured with a known reference surface, or standard. For our purposes, a useful "standard" is a sheet of white paper with an assumed reflectance of 0.8 . Some white paper has a chemical added that luminesces in the presence of ultraviolet radiation. The student is asked to devise an experiment to determine if the "standard" paper is subject to this effect, and select a paper that is free of luminescence for reflectance measurements in sunlight. We also have a limited quantity of Kodak gray cards that can be checked out. Now that a "standard" is available, the student is asked to devise a procedure to measure reflectance using both a natural source (sunlight) and an artificial source (tungsten lamp). When determining the reflectance, how does the radiance of the "standard" vary with solar incidence angle, and what should the orientation be for correct measurements? 
The radiometer is placed in Radiance Configuration I or II to determine the reflectance of various artificial surfaces in sunlight, such as paints of various colors, metals (Al foil), fabrics (various colors, light and dark), building materials (concrete vs. asphalt, different color bricks, etc.), cycle seats, whatever. Both the visible and the infrared filters are used. Several materials are then selected and measured using a tungsten lamp as the source to compare spectral characteristics. The radiometer is next used to determine the reflectance of natural surfaces in sunlight, such as bare soil, grass, ground cover, etc. We check to see if these surfaces are lambertian. Again, these measurements are done using both the visible and the infrared filters.

The radiometer is also used to determine the transmission of window materials by making measurements from both the inside and from the outside. House or apartment windows are good targets. A window that has a thermal control film is particularly interesting and may not have the same transmission in the visible and the infrared.

Another EXTRA CREDIT exercise is to devise a way with materials at hand to verify that the reflectance of the white paper "standard" is indeed 0.8 .

\section{Lab 7. The operational amplifier}

This lab involves the exploration of the basic DC properties of the operational amplifier (op-amp) and the characteristics of several circuits that can be built using it. A brief look at some common DC errors and noise concludes the lab. Each task explores a different circuit. Schematic diagrams for the circuits are given with suggested nominal component values and the ranges to explore. The experiments are:

1. inverting amplifier

2. follower

3. follower with gain

4. current source

5. transimpedance amplifier (TIA)

6. logarithmic converter

7. integrator (low-pass filter)

8. characterizing DC errors and noise (bipolar vs. FET)

\section{Lab 8. Interfacing PC detectors}

We now create an electronic interface (preamp) for a photoconductive (PC) cadmium sulfide detector and a thermistor (for use as a radiation detector or a thermometer). First, the DMM is used to measure resistance of the PC as a function of light level over an extended range. We plot the resistance vs. irradiance, using the radiometer with the Si detector as a reference. We determine the linearity of this over this extended irradiance range.

Knowing the current drawn by the PC detector from the previous step, we build a current source to furnish that current to the PC and observe the voltage drop across the detector vs. irradiance and compare with the ohmmeter readings obtained in the previous step. We change the temperature and see if the circuit behavior likewise changes.

Next we build a half-bridge using the $9 \mathrm{~V}$ batteries for the bias supply and an operating point and a matched load. This circuit is attempted in two configurations, (1) low-gain DC coupled and (2) high-gain AC coupled with a capacitor. 
Finally we build a full bridge with a differential amplifier configuration. An attempt is made to replace the $\mathrm{R}_{\mathrm{L}}$ leg of the bridge with the thermistor to achieve some degree of temperature compensation.

\section{Lab 9. Interfacing PV detectors}

In this lab, we demonstrate the two most effective circuits for interfacing a photovoltaic (PV) detector. We ignore the use of a simple voltmeter, ammeter or single load resistor as these are not widely applicable.

The follower is used to measure the open-circuit voltage $V_{o c}$ of the silicon photodiode. Then the transimpedance amplifier (TIA) is used to measure the short-circuit current $\mathrm{I}_{\mathrm{sc}}$ of the photodiode. In both cases we plot output vs. irradiance using $1 / \mathrm{r}^{2}$. We take a brief look at the temperature sensitivity of the PV detector, and inspect behavioral differences when reverse bias is used. Both the bipolar and the FET op-amp are used.

As an aside, we try other ersatz-PV devices, such as the 1N34A germanium diode, the LED's and the 1N914 silicon diode. Even though these are not normally considered as detectors, they are light-sensitive and can cause bizarre effects in circuits. We attempt to measure their responsivities and dark resistances. The LED's are particularly interesting inasmuch as they have recently been described by Mims (1992) as narrow-band detectors near their nominal emission wavelengths.

\section{Lab 10. Polarization in radiometry}

In this final lab we introduce the linear sheet polarizers. Part 1 uses a single polarizer and a bare silicon detector to determine the degree of linear polarization from a standard light bulb. Part 2 additionally introduces the infrared-transmitting and the visible-transmitting filters, and asks what can be inferred about either the source or the polarizer. Part 3 asks for a proof of Malus' law $\left(E=E_{o} \cos ^{2} \theta\right.$, where $\theta$ is the relative angle between two linear polarizers.) Part 4 explores reflection from a piece of flat glass as a function of angle, with either sunlight or a tungsten lamp as the source. Both the transmitted and reflected linear polarization are measured, and we attempt to determine the index of refraction of the glass from a determination of Brewster's angle. Part 5 inspects various He-Ne lasers to determine if any of them are polarized (some are and some aren't). Part 6 takes the radiometer and polarizer outdoors and looks for natural sources that display linear polarization. Included are the sun, skylight at various orientations with respect to the sun, grass as a function of direction, bricks, and concrete or asphalt paving, etc. 


\section{RADIOMETRY LABORATORY MANUAL}

A lab manual, separate from the course notes, has been prepared for student purchase. It incorporates the lab instructions, procedures, etc; the contents include:

Introductory material

The lab notebook; its function, contents and organization

Detailed instructions for each of the eleven laboratory experiments

At the end of the lab manual are several data sheets and application notes pertinent to the components used in the experiments. They include the following:

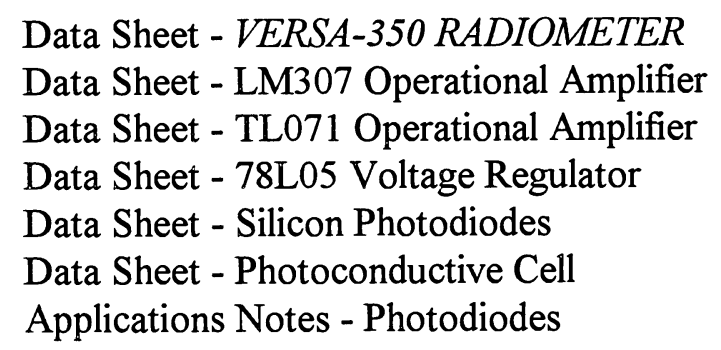

\section{NOTES FOR TEACHERS AND THE LAB NOTEBOOK}

The students are asked to record their procedures, observations, data and conclusions in a lab notebook. It need not be fancy, it may be spiral or 3-ring bound. It is turned in for grading at the end of the semester. The suggested format is shown in the Appendix. For multi-part experiments, it is desirable to keep the center section (procedure, data, processing and analysis) together for each part. A particularly useful component is the request for suggestions for improvement, identification of trouble spots, etc. These notebooks are turned in at the end of the semester for my assessment. The lab component of the total course grade is $25 \%$. Individual lab grades are weighted by the amount of work involved; i.e., Lab 1 is worth more than labs 2 and 3 . All furnished equipment is turned in at the end of the semester; with the course grade as ransom!

The students are encouraged to work with others setting up experiments, taking data, etc., The raw data obtained in this manner, along with graphs of the acquired data, are "community property." They may freely discuss the contents of the lab reports, but I ask that each student do their own data processing, analysis and write-up.

The eleven experiments are closely keyed with the class lecture schedule and are most useful when performed in order according to the timing suggested on the course syllabus. The students must be encouraged to avoid the misteak of putting it off until Thanksgiving or Spring break; there is way too much to do and they will invariably end up short on time, understanding and even sunshine. 


\section{CONCLUSIONS}

This take-home lab has been successfully conducted for the past five years for our juniors in the Optical Engineering program. Feedback from the students has in general been positive; most of the students feel that the labs are valuable, particularly when they are done in a timely manner coordinated with the lectures. There is a quite a bit of work involved, and I tend to add a couple of interesting tidbits each year. There is a great rush on my part at the end of the semester to get all of the reports read and graded, but I consider it well worth the effort. Keeping a good lab notebook is so important in the engineering and scientific community that positive reinforcement is worthwhile.

\section{REFERENCES}

Johnston, B.G. and J.M. Schroeer, "Take-home experiments for large classes," Phys. Teacher 30, 94 (1992).

Mims, Forrest, "Sun photometer with light-emitting diodes as spectrally sensitive detectors," Appl. Opt. 31, 6965 (1992).

APPENDIX - FORMAT FOR TAKE-HOME LAB EXPERIMENT REPORT

Name
Date
Take-home experiment \#

\section{TITLE}

Introduction: What do you wish to demonstrate, observe or measure? When, where and how was the experiment performed? Who assisted?

Procedures: Steps necessary to conduct the experiment. Equipment was required.

Data: Tabular form is best.

Data Processing: Include averaging, smoothing, calculations, transformations, etc. as required.

Analysis: Use graphs, uncertainty estimates, etc., where relevant.

Conclusions: Discussion, with lessons learned and suggestions for improvement. 